\title{
Sodium intake, cardiovascular disease, and physiology
}

\author{
Simon N. Thornton
}

I read with great interest the Review by He and MacGregor (Role of salt intake in prevention of cardiovascular disease: controversies and challenges. Nat. Rev. Cardiol. 15, 371-377; 2018) ${ }^{1}$ in which they put forward a very convincing case for populations in all countries of the world to decrease their salt - or rather sodium - intake in order to reduce the risk of cardiovascular disease, as demonstrated in the many clinical trials cited in the article. The Review is very comprehensive, but physiology seems to have been overlooked.

A. C. Guyton stated that "the control of sodium concentration in the intact state is accomplished mainly by the [antidiuretic hormone] $(\mathrm{ADH})$-thirst feedback mechanism" (REFS $\left.{ }^{2,3}\right)$. Furthermore, "increased salt intake results in increased thirst; therefore, a proportionate amount of water is consumed to match the salt" (REF. $\left.{ }^{4}\right)$. Guyton and colleagues talk about the need to drink water with sodium intake, but at no point in the Review by $\mathrm{He}$ and MacGregor is water intake mentioned. The appropriate physiological responses to increased plasma levels of sodium (that is, increased osmolality) are thirst and release of $\mathrm{ADH}$ (also known as vasopressin). A thirst-induced increase in fluid intake leads, initially, to an increase in blood volume, which persists until urine and sodium excretion return volume and osmolality to the normal range following reduced release of $\mathrm{ADH}$ and the sodium-retaining hormone aldosterone. Guyton's work was in dogs, but the regulation of hydromineral balance is not known to differ between mammalian species, especially between rats and dogs (in which much work has been performed to understand the mechanisms of this complex regulation) and humans ${ }^{5}$.

A clinical trial showed that a diet high in salt normally increases blood pressure and decreases plasma renin activity and aldosterone levels in the blood and urine ${ }^{6}$. Similar results with appropriate fluid intake have been observed in rats ${ }^{7}$. However, in humans, increased fluid intake does not seem to occur with increased sodium intake, and this hypohydration affects both the intracellular and especially the extracellular fluid compartments, the latter being associated with hypovolaemia. The physiological mechanisms regulating decreased volume are thirst; $\mathrm{ADH}$, angiotensin, and aldosterone release; and salt appetite mediated by a central synergistic action of the released angiotensin and aldosterone ${ }^{8}$. These two hormones continue to be released and salt consumed unless blood volume is restored by increased drinking.

Interestingly, the majority of medications used to combat cardiovascular disease are blockers of the renin-angiotensin system $^{9}$ and, more recently, antagonists of aldosterone $\mathrm{e}^{10}$. However, according to normal physiology, increased salt intake should already have decreased the levels of these two hormones. Knowing that the physiology of hydromineral balance regulation is similar in most mammals, this observation suggests that humans are chronically, but mildly, hypohydrated.

In conclusion, physiological regulation suggests that no matter how much dietary sodium intake is reduced, angiotensin and aldosterone will continue to be released and salt intake and cardiovascular disease propagated - unless blood volume is restored. The part of physiological regulation that is often overlooked is that blood volume can very effectively be restored by drinking appropriate quantities of water.

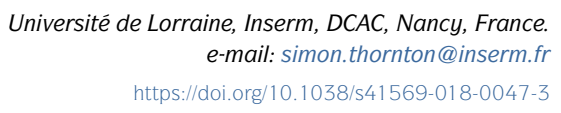

Université de Lorraine, Inserm, DCAC, Nancy, France. e-mail:simon.thornton@inserm.fr https://doi.org/10.1038/s41569-018-0047-3

Simon N. Thornton

1. He, F. J. \& MacGregor, G. A. Role of salt intake in prevention of cardiovascular disease: controversies and challenges. Nat. Rev. Cardiol. 15, 371-377 (2018).

2. Young, D. B., Pan, Y. J. \& Guyton, A. C. Control of extracellular sodium concentration by antidiuretic hormone-thirst feedback mechanism. Am. J. Physiol. 232, R145-R149 (1977).

3. Guyton, A. C. et al. Integration and control of circulatory function. Int. Rev. Physiol. 9, 341-385 (1976).

4. Guyton, A. C. Blood pressure control - special role of the kidneys and body fluids. Science $\mathbf{2 5 2}$, 1813-1816 (1991).

5. Thornton, S. N. Thirst and hydration: physiology and consequences of dysfunction. Physiol. Behav. 100, 15-21 (2010).

6. Garg, R. et al. Low-salt diet increases insulin resistance in healthy subjects. Metabolism 60, 965-968 (2011).

7. Stocker, S. D. et al. Elevated dietary salt suppresses renin secretion but not thirst evoked by arterial hypotension in rats. Am. J. Physiol. Regul. Integr. Comp. Physiol. 284, R1521-R1528 (2003).

8. Zhang, D. M., Stellar, E. \& Epstein, A. N Together intracranial angiotensin and systemic mineralocorticoid produce avidity for salt in the rat. Physiol. Behav. 32, 677-681 (1984).

9. Taler, S. J. Initial treatment of hypertension. N. Engl. J. Med. 378, 1953-1954 (2018).

10. Zannad, F. et al. Eplerenone in patients with systolic heart failure and mild symptoms. N. Engl. J. Med. 364, 11-21 (2011).

\section{Reply to 'Salt intake, cardiovascular disease, and physiology'}

\section{Feng J. He and Graham A. MacGregor}

We are grateful to Simon Thornton for his Correspondence (Salt intake, cardiovascular disease, and physiology. Nat. Rev. Cardiol. https://doi.org/10.1038/s41569-018-0047-3; 2018) ${ }^{1}$ on our Review (Role of salt intake in prevention of cardiovascular disease: controversies and challenges. Nat. Rev. Cardiol. 15, 371-377; 2018) ${ }^{2}$. However, our Review was focused on the evidence that salt intake is a major determinant of population blood pressure, not on the mechanisms by which salt can cause high blood pressure.

We and others have published several papers on the potential mechanisms ${ }^{3-5}$ but we did not think that this information was directly relevant to public health. When humans consume more salt, plasma levels of sodium and chloride increase, which causes increased thirst and fluid consumption. At the same time, this increase in the sodium and chloride concentrations increases extracellular fluid volume. Under normal circumstances, this increase in extracellular volume suppresses the renin-angiotensinaldosterone system and also increases release of atrial natriuretic peptide (ANP), which to some extent mitigates the rise in blood pressure that would have occurred. However, long-term small increases in extracellular or, more accurately, intrathoracic - volume slowly raise blood pressure.

In studies that we and others have performed, when individuals increase their 